\title{
A importância do plano de cuidados para pacientes oncológicos em cuidados paliativos desenvolvidos pelos enfermeiros da Estratégia Saúde da Família
}

The importance of the care plan for cancer patients in palliative care developed by nurses from the Family Health Strategy

La importancia del plan de cuidados al paciente oncológico en cuidados paliativos desarrollado por enfermeros de la Estrategia Salud de la Familia

Recebido: 18/01/2021 | Revisado: 21/01/2021 | Aceito: 22/01/2021 | Publicado: 28/01/2021

Camila Stefani Lomanto dos Santos ORCID: https://orcid.org/0000-0001-5580-7061 NOVA Faculdade, Brasil

E-mail: camilalomanto@ outlook.com

Fernanda Penido Matozinhos

ORCID: https://orcid.org/0000-0003-1368-4248 Universidade Federal de Minas Gerais, Brasil E-mail: nandapenido@hotmail.com

Laydson Adrian de Araújo ORCID: ttps://orcid.org/0000-0001-9082-2873 NOVA Faculdade, Brasi

E-mail: laydsonaraujo@novafaculdade.com.br

Thales Philipe Rodrigues da Silva ORCID: https://orcid.org/0000-0002-7115-0925

Universidade Federal de Minas Gerais, Brasil E-mail: thalesphilipe27@ hotmail.com

\begin{abstract}
Resumo
Objetivo: O presente estudo objetivou descrever a importância do plano de cuidados na assistência de enfermagem ao paciente oncológico que necessita de cuidados paliativos realizado pelos profissionais da Estratégia Saúde da Família. Métodos: Estudo de revisão integrativa da literatura, desenvolvida por meio de pesquisa sistemática na base de dados PUBMED e BVS. A revisão bibliográfica se restringiu às publicações dos últimos cinco anos. A análise dos resultados foi feita de forma crítica, finalizando o processo de inclusão e exclusão das obras que não se encaixam com o tema proposto, garantindo a validação da revisão e das obras selecionadas. Resultados: Os resultados encontrados ressaltam que o enfermeiro oferece assistência que conferem meios que propiciam um enfrentamento eficaz da doença, por meio da redução dos impactos dos sintomas nos pacientes oncológicos. Conclusão: Evidenciou-se a importância do Enfermeiro nos cuidados paliativos domiciliares, que atua oferecendo uma assistência pautada na humanização, conforto e dignidade durante o processo de terminalidade da vida do individuo.
\end{abstract}

Palavras-chave: Cuidados de enfermagem; Assistência domiciliar; Cuidados paliativos; Oncologia; Estratégia saúde da família.

\begin{abstract}
Objective: The present study aimed to describe the importance of the care plan in nursing care for cancer patients who need palliative care performed by professionals in the Family Health Strategy. Methods: Study of integrative literature review, developed through systematic research in the PUBMED and VHL database. The bibliographic review was restricted to publications from the last five years. The analysis of the results was done critically, ending the process of inclusion and exclusion of works that do not fit the proposed theme, ensuring the validation of the review and selected works. Results: The results found emphasize that the nurse offers assistance that provides means that provide an effective coping with the disease, by reducing the impact of symptoms on cancer patients. Conclusion: The importance of the nurse in palliative care at home was evidenced, who works by offering assistance based on humanization, comfort and dignity during the individual's terminal life process.
\end{abstract}

Keywords: Nursing care; Home nursing; Palliative care; Medical oncology; Family health strategy.

\section{Resumen}

Objetivo: El presente estudio tuvo como objetivo describir la importancia del plan de cuidados en la atención de enfermería para pacientes con cáncer que necesitan cuidados paliativos realizados por profesionales de la Estrategia Salud de la Familia. Métodos: Estudio de revisión integradora de la literatura, desarrollado a través de una 
investigación sistemática en la base de datos PUBMED y BVS. La revisión bibliográfica se restringió a publicaciones de los últimos cinco años. El análisis de los resultados se realizó de manera crítica, finalizando el proceso de inclusión y exclusión de trabajos que no se ajustan a la temática propuesta, asegurando la validación de la revisión y los trabajos seleccionados. Resultados: Los resultados encontrados enfatizan que la enfermera ofrece asistencia que brinda medios que brindan un afrontamiento efectivo de la enfermedad, al reducir el impacto de los síntomas en los pacientes con cáncer. Conclusión: se evidenció la importancia de la enfermera en los cuidados paliativos en el hogar, que trabaja ofreciendo asistencia basada en la humanización, el confort y la dignidad durante el proceso de vida terminal del individuo.

Palabras clave: Atención de enfermería; Atención domiciliaria de salud; Cuidados paliativos; Oncología médica; Estrategia de salud familiar.

\section{Introdução}

O câncer pode ser causado por diferentes fatores de risco e de causas múltiplas como os fatores ambientais, culturais, socioeconômicos, por estilos de vida, hábitos de fumar e alimentares, fatores genéticos e o próprio processo de envelhecimento (M. M. De Oliveira, Malta, Guauche, de Moura, \& Azevedo e Silva, 2015).Diante disso, as neoplasias têm crescido em todo o mundo e ocupam a segunda causa de morte na maioria dos países da Europa e américa do norte. Já no Brasil, em 2011, o câncer foi responsável por 16,4\% dos óbitos, sendo a segunda causa de mortalidade (Malta et al., 2014).

Neste contexto, o câncer pode apresentar diversos sinais e sintomas como a dor, náuseas e vômitos, constipação, que estão entre os sintomas mais comuns que causam sofrimento ao paciente. Diante disso, as ações para controle do câncer não devem se basear somente às ações de prevenção, à detecção precoce, ou até mesmo somente ao diagnóstico e tratamento, mas deve considerar também os cuidados paliativos (INCA, 2020).

Segundo a Organização Mundial da Saúde (OMS) o cuidado paliativo é uma abordagem que visa melhorar a qualidade de vida de pacientes e familiares no enfrentamento a doenças terminais, por meio da prevenção e do alívio do sofrimento pelo diagnóstico precoce, avaliação e tratamento da dor e outros problemas físicos, psicossociais e espirituais (World Health Organization, 2007)(Costa de Oliveira, 2019). Buscam ainda, conduzir ações que objetivam reduzir os sintomas e as aflições, além de ajudar na autonomia e ampliar o suporte aos familiares e cuidadores (Marcucci, Perilla, Brun, \& Cabrera, 2016).

Neste contexto dos cuidados paliativos nos pacientes portadores de neoplasias malignas, a atenção primária à saúde (APS) tem o poder de influenciar nas condições determinantes de algumas doenças, além de ampliar e coordenar o acesso aos cuidados de saúde nas diferentes fases da vida da população, incluindo o paciente com neoplasias malignas. Propõe ainda, atenção integral do indivíduo bem como, a família e coletividade, incluindo assim, a proximidade da morte e o acompanhamento em todo o processo (Marcucci et al., 2016).

Sendo assim, APS guia-se pelos princípios da coordenação do cuidado, do vínculo e continuidade, da integralidade, da responsabilização, da humanização, da equidade e da participação social, sendo de suma importância a aplicação desses princípios e definições que exigem incorporar os cuidados paliativos dentro da estratégia saúde da família (ESF)(Combinato \& Ferreira Martins, 2012).

Diante do aumento de casos de câncer e da influência das doenças crônicas não transmissíveis (DCNTs) na mortalidade da população brasileira e ainda associada à maior longevidade, esses fatores acarretam uma demanda crescente sobre o sistema de saúde (Malta et al., 2017). Com a evolução do quadro clínico, o paciente pode apresentar uma perda gradativa da autonomia, fazendo com que necessite de cuidados contínuos e causando um prolongado sofrimento até o óbito (Marcucci et al., 2016). Diante deste cenário, é possível observar que há dificuldade do enfermeiro em enfrentar situações com este tipo de paciente, pois a prática dos cuidados paliativos, em alguns aspectos, ainda não está introduzida à rotina de cuidados de muitos profissionais (Crenças et al., 2017). (9).

Logo, percebe-se que o enfermeiro é de extrema importância nesse contexto, visto ser este o único profissional com 
real qualificação para elaborar o plano de cuidados direcionado as necessidades a serem trabalhadas de cada paciente oncológico e de seus familiares, promovendo assim maior conforto e segurança nesse processo de morte. Diante das diferentes abordagens pesquisadas, justifica-se a importância deste estudo, pela deficiência de estudos e publicações científicas na área de cuidados paliativos domiciliares, todavia, quanto à percepção e participação do enfermeiro inserido nesse contexto. Justifica-se também, pela necessidade de se aproximar desta realidade, por meio de pesquisas e conhecer as demandas de cuidado específicas deste grupo; e de se propor uma reflexão acerca do trabalho desenvolvido pela ESF, baseado nos princípios de integralidade e interdisciplinaridade.

O presente estudo objetivou descrever a importância do plano de cuidados na assistência de enfermagem ao paciente oncológico que necessita de cuidado paliativo realizado pelos profissionais da Estratégia Saúde da Família.

\section{Metodologia}

Revisão integrativa da literatura (Pereira, Shitsuka, Parreira, \& Shitsuka, 2018), desenvolvida por meio de pesquisa sistemática na base de dados informatizadas da Biblioteca Virtual em Saúde do Ministério da Saúde (BVSMS) e MEDLINE (Pubmed). A revisão integrativa é um método de investigação científica que utiliza avaliação criteriosa de dados obtidos de diferentes estudos primários. Permite ainda, a identificação de lacunas de determinado tema e possibilita fundamentação para futuras investigações, além de auxiliar a sintetização da evidência já disponível na literatura sobre determinado assunto (Ercole, Melo, \& Alcoforado, 2014).

Essa revisão foi realizada seguindo as seguintes etapas: desenvolvimento da pergunta norteadora; busca dos estudos primários nas bases de dados; extração de dados dos estudos; avaliação dos estudos selecionados; análise e síntese dos resultados e apresentação da revisão (L. M. M. De Sousa, Firmino, Marques-Vieira, Severino, \& Pestana, 2018).

Para realizar a busca nas bases de dados, a pergunta norteadora foi elaborada segundo a estratégia PICO (Patient/population/disease; Intervention or issue ofinterest; Comparison, Intervention or issue of interest; Outcome) (Quadro 1). A População foi definida como "pacientes oncológicos", Intervenção como "cuidados paliativos", Comparação não houve e Resultado não houve. Assim, a pergunta de pesquisa foi apresentada como: "Qual a importância do plano de cuidados para indivíduos portadores de neoplasias em cuidados paliativos desenvolvido pelos profissionais da estratégia em saúde da família?".

Quadro 1 - Estratégia PICo, 2020.

\begin{tabular}{|l|l|l|}
\hline \multicolumn{3}{|c|}{ Estratégia PICo } \\
\hline PICo & Variáveis & Componentes \\
\hline P & População & Pacientes oncológicos \\
\hline I & Interesse & Cuidados paliativos \\
\hline Co & Contexto & Atenção Primária a Saúde \\
\hline
\end{tabular}

Fonte: Autores.

A chave de busca para as bases de dados pesquisadas (BVS e PUBMED) foi elaborada utilizando as palavras-chave cuidados paliativos AND cuidado domiciliar AND cuidados de enfermagem. A revisão bibliográfica se restringiu às publicações dos últimos cinco anos (entre 2015 a 2020) e foi realizada em outubro de 2020.

A análise dos resultados foi feita de forma crítica, finalizando o processo de inclusão e exclusão das obras que não se encaixam com o tema proposto, garantindo a validação da revisão e das obras selecionadas. 
No momento final foi realizada a interpretação dos dados a partir da fundamentação dos resultados da avaliação crítica, traçando um diálogo entre os autores, as obras e documentos em busca de uma compreensão sobre a importância do plano de cuidados na assistência de enfermagem ao paciente oncológico que necessita de cuidados paliativos no realizados pela ESF.

\section{Resultados}

A estratégia de busca inicial gerou um total de 961 artigos que potencialmente respondiam a pergunta de pesquisa por meio da pesquisa bibliográfica. Após a exclusão de duplicatas (170), 11 estudos preencheram os critérios de inclusão e foram incluídos na revisão integrativa (Figura 1). As características dos estudos incluídos na revisão estão resumidas na Tabela 1.

Figura 1 - Fluxograma, de seleção dos estudos selecionados

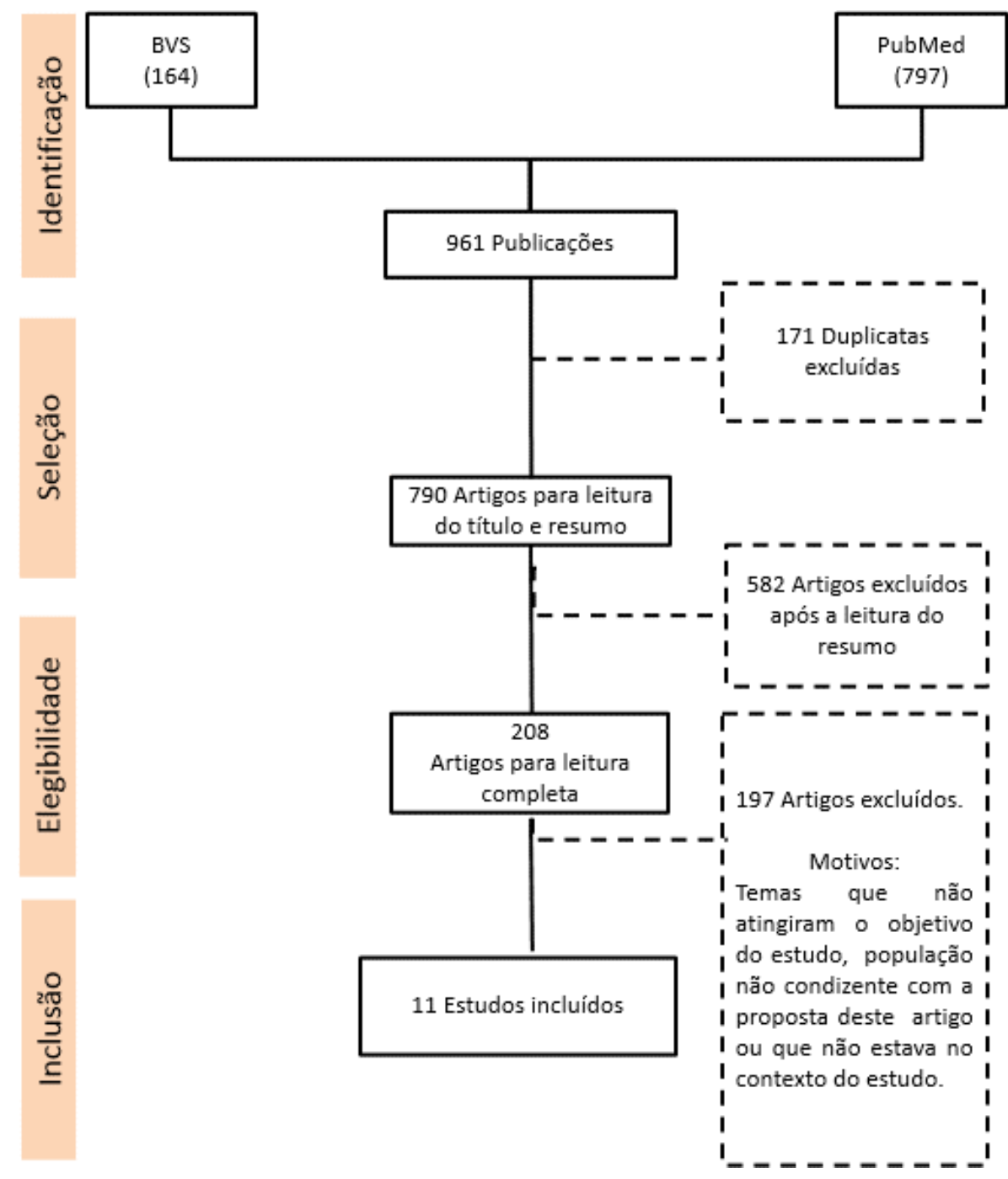

Fonte: Autores.

Entre os 11 (Maetens et al., 2019)(M. B. P. Oliveira et al., 2017)(Passarelles, Rios, \& Santana, 2019)(Martins, Correa Júnior, Santana, \& Santos, 2018)(Hey, Hermann, Mercês, \& Lacerda, 2017)(Justino, Kasper, Santos, Quaglio, \& Fortuna, 2020)(Pilatti et al., 2017)(Heydari, Hojjat-Assari, Almasian, \& Pirjani, 2019)(Matos, Muniz, Viegas, Przylynski, \& Holz, 
2016)(Andrande, Costa, Costa, Santos, \& Brito, 2017)(J. M. Sousa \& Alves, 2015) artigos analisados, os mesmos foram publicados entre os anos de 2015 a 2020 (Tabela 1). Os países de realização dos artigos foram Bélgica, Brasil e Irã (Tabela 1).

Tabela 1 - Características dos estudos incluídos na revisão integrativa.

\begin{tabular}{|c|c|c|c|c|}
\hline $\begin{array}{l}\text { Nome do } \\
\text { primeiro } \\
\text { autor }\end{array}$ & Título & $\begin{array}{l}\text { Ano/Local } \\
\text { de estudo }\end{array}$ & Delineamento & Principais resultados \\
\hline $\begin{array}{l}\text { Arno } \\
\text { Maetens }\end{array}$ & $\begin{array}{l}\text { Impact of palliative home } \\
\text { care support on the quality } \\
\text { and costs of care at the end } \\
\text { of life: a population-level } \\
\text { matched cohort study }\end{array}$ & $\begin{array}{c}2019 \\
\text { Bélgica }\end{array}$ & $\begin{array}{l}\text { Estudo de coorte } \\
\text { em nível } \\
\text { populacional }\end{array}$ & $\begin{array}{l}\text { O uso do apoio à atenção domiciliar paliativo } \\
\text { impacta positivamente a qualidade do cuidado } \\
\text { e reduz os custos totais de cuidados no final } \\
\text { da vida na Bélgica. }\end{array}$ \\
\hline Heshmatolah & $\begin{array}{l}\text { Exploring health care } \\
\text { providers' perceptions about } \\
\text { home-based palliative care } \\
\text { in terminally ill cancer } \\
\text { patients }\end{array}$ & $\begin{array}{c}2019 \\
\text { Irã }\end{array}$ & Estudo qualitativo & $\begin{array}{l}\text { A modificação estrutural e de processos na } \\
\text { assistência à saúde pode proporcionar } \\
\text { condições em que pacientes com câncer } \\
\text { terminal recebam cuidados adequados em casa } \\
\text { e experimentem a morte com dignidade por } \\
\text { meio do apoio da família, amigos e cuidados de } \\
\text { saúde. }\end{array}$ \\
\hline $\begin{array}{l}\text { Eveline } \\
\text { Treméa } \\
\text { Justino }\end{array}$ & $\begin{array}{l}\text { Cuidados paliativos na } \\
\text { atenção primária à saúde: } \\
\text { revisão de escopo }\end{array}$ & $\begin{array}{l}2020 \\
\text { Brasil }\end{array}$ & Estudo de revisão & $\begin{array}{l}\text { Evidenciou-se que os cuidados paliativos na } \\
\text { atenção primária à saúde vêm sendo } \\
\text { gradativamente desenvolvidos, mas há que se } \\
\text { considerar o modo de organização da atenção } \\
\text { primária à saúde e as políticas sociais que as } \\
\text { sustentam ou enfraquecem, sendo } \\
\text { considerado um desafio complexo. }\end{array}$ \\
\hline Martins & $\begin{array}{l}\text { Corporeidade de adoecidos } \\
\text { oncológicos em cuidados } \\
\text { paliativos domiciliares: a } \\
\text { vivência de familiares }\end{array}$ & $\begin{array}{c}2018 \\
\text { Brasil }\end{array}$ & Pesquisa qualitativa & $\begin{array}{l}\text { O cuidado está compartimentalizado para os } \\
\text { familiares que contrapõem os préstimos ao } \\
\text { corpo social e préstimos ao corpo físico, } \\
\text { recordando primeiramente do último. }\end{array}$ \\
\hline $\begin{array}{l}\text { Cristiani } \\
\text { Garrido de } \\
\text { Andrade }\end{array}$ & $\begin{array}{l}\text { Cuidados paliativos e } \\
\text { comunicação: estudo com } \\
\text { profissionais de saúde do } \\
\text { serviço de atenção } \\
\text { domiciliar }\end{array}$ & $\begin{array}{l}2017 \\
\text { Brasil }\end{array}$ & Pesquisa qualitativa & $\begin{array}{l}\text { Conclui-se que a comunicação configura-se } \\
\text { como um componente fundamental do } \\
\text { cuidado com o paciente e é de suma } \\
\text { relevância para a promoção dos cuidados } \\
\text { paliativos. }\end{array}$ \\
\hline $\begin{array}{c}\text { Maria do } \\
\text { Bom Parto de } \\
\text { Oliveira }\end{array}$ & $\begin{array}{l}\text { Atendimento domiciliar } \\
\text { oncológico: percepção de } \\
\text { familiares/cuidadores sobre } \\
\text { cuidados paliativos }\end{array}$ & $\begin{array}{c}2017 \\
\text { Brasil }\end{array}$ & $\begin{array}{l}\text { Pesquisa } \\
\text { qualitativa }\end{array}$ & $\begin{array}{l}\text { Foi possível resgatar a importância dos } \\
\text { cuidados paliativos no domicílio como } \\
\text { possibilidade de minimizar o impacto do } \\
\text { sofrimento com a doença e assegurar a } \\
\text { dignidade no processo de morrer. }\end{array}$ \\
\hline
\end{tabular}




\begin{tabular}{|c|c|c|c|c|}
\hline $\begin{array}{l}\text { Patrícia } \\
\text { Pilatti }\end{array}$ & $\begin{array}{l}\text { Cuidado paliativos } \\
\text { oncológicos em um serviço } \\
\text { público de } \quad \text { atenção } \\
\text { domiciliar }\end{array}$ & $\begin{array}{c}2017 \\
\text { Brasil }\end{array}$ & Estudo transversal & $\begin{array}{l}\text { O presente estudo permitiu a identificação das } \\
\text { principais demandas dos pacientes em } \\
\text { cuidados paliativos oncológicos atendidos por } \\
\text { um serviço de Atenção Domiciliar. Observa- } \\
\text { se na prática deste serviço que a maioria dos } \\
\text { pacientes apresentou necessidade de cuidados } \\
\text { complexos, mas possíveis de serem realizados } \\
\text { no domicílio pela equipe de saúde e/ou } \\
\text { cuidador capacitado. }\end{array}$ \\
\hline $\begin{array}{c}\text { Albimara } \\
\text { Hey }\end{array}$ & $\begin{array}{l}\text { Participação da enfermeira } \\
\text { nos cuidados paliativos } \\
\text { domiciliares }\end{array}$ & $\begin{array}{c}2017 \\
\text { Brasil }\end{array}$ & Pesquisa qualitativa & $\begin{array}{l}\text { Concluiu-se que a presença do enfermeiro } \\
\text { nessa modalidade de cuidado é fundamental, } \\
\text { podendo contribuir para a estruturação desse } \\
\text { cuidado no Sistema Único de Saúde do Brasil. }\end{array}$ \\
\hline $\begin{array}{l}\text { Michele } \\
\text { Rodrigues } \\
\text { Matos }\end{array}$ & $\begin{array}{l}\text { Significado da atenção } \\
\text { domiciliar e o momento } \\
\text { vivido pelo paciente } \\
\text { oncológico em cuidados }\end{array}$ & $\begin{array}{c}2016 \\
\text { Brasil }\end{array}$ & $\begin{array}{l}\text { Pesquisa } \\
\text { qualitativa }\end{array}$ & $\begin{array}{l}\text { Conclui-se que as potencialidades deste } \\
\text { modelo de atenção vão ao encontro da } \\
\text { assistência que visa contemplar os princípios } \\
\text { do cuidado paliativo, como o bem-estar e o } \\
\text { estar melhor desses pacientes. }\end{array}$ \\
\hline $\begin{array}{c}\text { Janaina } \\
\text { Meirelles } \\
\text { Sousa }\end{array}$ & $\begin{array}{l}\text { Competências do enfermeiro } \\
\text { para o cuidado paliativo na } \\
\text { atenção domiciliar }\end{array}$ & $\begin{array}{c}2015 \\
\text { Brasil }\end{array}$ & Estudo exploratório & $\begin{array}{l}\text { O elenco de competências apresentou } \\
\text { confiabilidade interna e fornece afirmações } \\
\text { assertivas sobre a atuação do enfermeiro em } \\
\text { cuidado paliativo na atenção domiciliar. }\end{array}$ \\
\hline $\begin{array}{c}\text { Dayana } \\
\text { Medeiros } \\
\text { do Amaral } \\
\text { Passarelles }\end{array}$ & $\begin{array}{l}\text { Diagnósticos de enfermagem } \\
\text { em cuidados paliativos } \\
\text { oncológicos: revisão } \\
\text { integrativa }\end{array}$ & $\begin{array}{c}2019 \\
\text { Brasil }\end{array}$ & Revisão Integrativa & $\begin{array}{l}\text { Ao identificar uma diversidade de } \\
\text { diagnósticos de enfermagem ocorrentes em } \\
\text { pacientes em cuidados paliativos oncológicos, } \\
\text { conclui-se que se faz necessário a construção } \\
\text { de um diagnóstico de enfermagem de } \\
\text { síndrome, para otimizar o raciocínio clínico } \\
\text { dos enfermeiros em cuidados paliativos. }\end{array}$ \\
\hline
\end{tabular}

Fonte: Autores.

Os artigos são unanimes em reconhecer que os enfermeiros são capazes de oferecer assistências eficazes ao paciente oncológico inserido na ESF através dos cuidados paliativos domiciliares, que tendem a minimizar o sofrimento na terminalidade de vida, além de aumentar a qualidade de vida destes pacientes. Entre esses artigos, Maetens et al., (2019)(Maetens et al., 2019) e Oliveira et al., (2017)(M. B. P. Oliveira et al., 2017), avaliaram o custo- efetividade dos cuidados paliativos domiciliares e mencionam que impactam positivamente ao cuidado prestado.

Sobre a importância da comunicação Andrade et al., (2017)(Andrande et al., 2017) e Matos et al., (2016)(Matos et al., 2016) observaram que a comunicação é de suma relevância para o cuidado com o paciente e se configura como um componente fundamental para a promoção dos cuidados paliativos, além de servir como instrumento de trabalho para os multiprofissionais ali envolvidos.

Também é relatado por Heydari et al., (2019) (Heydari et al., 2019), Martins et al., (2018)(Martins et al., 2018) e Oliveira et al., (2017)(M. B. P. Oliveira et al., 2017) as percepções dos cuidadores relacionado aos cuidados paliativos domiciliares e que diante do sofrimento causado ao paciente oncológico em fase terminal, esse tipo de assistência possibilita 
minimizar o impacto vivenciado pela doença. Além de assegurar a dignidade no processo de morrer por meio do apoio da família, amigos e cuidados de saúde.

Em relação à atuação do enfermeiro, Passarelles et al., (2019)(Passarelles et al., 2019), Hey et al., (2017)(Hey et al., 2017) e Sousa et al., (2015)(J. M. Sousa \& Alves, 2015) citam que o enfermeiro apresenta competências e confiabilidade nessa modalidade de cuidado, e sua presença é fundamental, podendo contribuir para a estruturação desse cuidado no Sistema Único de Saúde do Brasil.

Outro fator avaliado por Passarelles et al., (2019)(Passarelles et al., 2019), Pilatti et al., (2017)(Pilatti et al., 2017), Matos et al., (2016)(Matos et al., 2016) e Justino et al., (2020)(Justino et al., 2020) foi a efetividade do cuidado paliativo na APS. Os autores evidenciam que os cuidados paliativos desenvolvidos na APS vêm sendo gradativamente desenvolvidos e incorporados, entretanto as principais demandas dos pacientes oncológicos em cuidados paliativos são atendidas pela Atenção Domiciliar pela equipe de saúde e/ou cuidador capacitado.

\section{Discussão}

Essa revisão integrativa evidenciou que a presença do enfermeiro nos cuidados paliativos domiciliares é de extrema importância, pois as condutas de enfermagem conferem meios que principiam um enfrentamento eficaz da doença.

Os cuidados paliativos domiciliares reduzem os impactos dos sintomas nesses pacientes, e minimiza o impacto do sofrimento com a doença, além de assegurar a dignidade no processo de morte. E mesmo sendo uma assistência de alto custo, evidenciou-se que os cuidados paliativos domiciliares obtiveram custos totais médios mais baixos em comparação ao suporte de cuidados paliativos hospitalares(M. B. P. Oliveira et al., 2017)(Martins et al., 2018), mostrando ser a opção com melhor custo benefício para o paciente e familiar.Dessa forma, é necessário que os familiares de pacientes com o diagnóstico de câncer mantenham forte vínculo com a equipe de saúde, sendo o aliado principal no processo do cuidado em domicílio e de enfrentamento da doença terminal(Passarelles et al., 2019).

Diante deste contexto, é possível observar que o cuidado paliativo oncológico é um cenário de assistência que está em construção e, a enfermagem vem oferecendo cuidados importantes a essa população. Por meio da padronização de linguagem o profissional de enfermagem deve ter um raciocínio clínico perspicaz para identificar as respostas humanas de alta complexidade que esse indivíduo possa demandar (Martins et al., 2018).

Diante do exposto, é notório que além de prestar a maioria dos cuidados, os enfermeiros são capazes de potencializar o planejamento da assistência no gerenciamento de sinais e sintomas, otimizando a prática clínica com a identificação do conjunto de sintomas em um único diagnóstico apropriado para o cuidado paliativo (Hey et al., 2017). Esses profissionais são essenciais para o paciente oncológico, tornando-se indiscutível direcionar continuamente a prática da enfermagem para os benefícios da população (Hey et al., 2017).

Desse modo, as condutas de enfermagem precisam conferir meios que principiam um enfrentamento eficaz da doença, além de oferecer suporte biopsicossocial e emocional a todos os envolvidos (Hey et al., 2017) . Ao realizarem os cuidados paliativos no domicílio, o enfermeiro deve conhecer o histórico clínico e atual do paciente e posteriormente, por meio de visita domiciliar, o ambiente onde vão prestar os cuidados, identificando suas potencialidades e fragilidades. Pois ao identificar as necessidades humanas do paciente no domicílio, o enfermeiro consegue fazer um diagnóstico, tornando-se possível realizar seu planejamento de cuidados, que deve ser reavaliado continuamente de acordo com a evolução do paciente (Justino et al., 2020).

Além disso, é de suma importância que o profissional envolvido nos cuidados de enfermagem saiba como utilizar as ferramentas essenciais para a assistência domiciliar, como a Escala de Avaliação de Sintomas de Edmonton que tem como objetivo avaliar os sintomas comuns em pacientes com câncer e em cuidados paliativos; a Escala de Performance de Karnofsky que é responsável por classificar os pacientes de acordo com o grau de deficiências funcionais, podendo também ser utilizada 
para avaliar a efetividade de diferentes terapias; e a Escala de Performance Paliativa que serve para acompanhar a curva evolutiva da doença, de forma a contribuir positivamente na tomada de decisão, previsão de prognóstico e definição de terminalidade. Todas essas escalas auxiliam na orientação do plano de cuidados específicos para cuidados paliativos, além de melhorar a gestão do atendimento (Pilatti et al., 2017).

Observa-se que na prática do cuidado paliativo domiciliar a maioria dos pacientes apresentou necessidade de cuidados complexos, pois esses cuidados não se resumem à execução de técnicas, exigindo do profissional atuação integral, humanizadora e compartilhada. A colaboração interprofissional e intersetorial na prestação de serviços de saúde é uma necessidade inevitável (Heydari et al., 2019). É imprescindível que os profissionais de saúde e as várias instituições tenham estreita coordenação e cooperação interdisciplinar e intersetorial para promover a saúde da comunidade (Matos et al., 2016). Os profissionais devem manter a comunicação como parte da assistência de cuidados paliativos, pois é um instrumento para promoção da humanização da assistência, tornando não só a equipe, mas também os familiares e o paciente (Andrande et al., 2017).

O enfermeiro é responsável por proporcionar conforto nesse momento difícil, minimizando seus medos e anseios, por meio do planejamento de ações a fim de controlar os impactos do câncer, tendo a sensibilidade de identificar desordens do campo físico, psíquico, social e espiritual (J. M. Sousa \& Alves, 2015). Uma das funções essenciais dos cuidados paliativos é a assistência integral aos cuidadores familiares, desde o diagnóstico até o avançar da doença e a morte, o enfermeiro fica próximo da família, ocorrendo um envolvimento de ambas as partes e a criação de vínculo, que não pode ser interrompido abruptamente após a morte do paciente (Pilatti et al., 2017).

É possível observar que existe uma limitação da atuação do enfermeiro e da interdisciplinaridade na atenção domiciliar dentro do contexto da ESF, reforçando a necessidade de preparação específica para se garantir adequada implementação dos cuidados paliativos neste cenário, o que pode indicar a necessidade de aquisição de novos conhecimentos acerca dos cuidados paliativos para manter e desenvolver suas próprias competências profissionais (J. M. Sousa \& Alves, 2015).

\section{Conclusão}

Por meio desse estudo, foi possível evidenciar a importância da enfermagem nos cuidados paliativos domiciliares, que atua oferecendo uma assistência pautada na humanização, conforto e dignidade no processo de terminalidade da vida.

O planejamento do plano de cuidados paliativos ao paciente oncológico se faz por meio do diagnóstico, identificando as necessidades e reconhecendo o contexto no qual o paciente está inserido, direcionando a assistência de enfermagem a fim de minimizar o sofrimento. Além de possibilitar a criação de laços entre o paciente, família e enfermeiro e vivenciando momentos significados na realização do cuidado. A pesquisa evidencia ainda, que existe uma deficiência em estudos específicos sobre a atuação do enfermeiro e elaboração do plano de cuidados para o paciente em cuidado paliativo.

Diante do exposto, esse trabalho evidência a necessidade de se reavaliar a abordagem dos temas relacionados ao processo de terminalidade da vida e sobre o cuidado paliativo, em especial no âmbito da ESF, durante a formação do enfermeiro. Ressalta-se a necessidade que novos estudos que vislumbrem o papel do Enfermeiro da ESF para a realização do plano de cuidados direcionado as necessidades do paciente oncológico em cuidados paliativos no domicilio e, consequentemente de seus familiares.

\section{Referências}

Andrande, C. G. de., Costa, S. F. G. da., Costa, I. C. P., Santos, K. F. O. dos., \& Brito, F. de M. (2017). Cuidados paliativos e comunicação: estudo com profissionais de saúde do serviço de atenção domiciliar. Revista de Pesquisa: Cuidado é Fundamental Online, 9(1), 215. https://doi.org/10.9789/21755361.2017.v9i1.215-221 
Combinato, D. S., \& Ferreira Martins, S. T. (2012). (Em defesa dos) Cuidados Paliativos na Atenção Primária à Saúde. Mundo Da Saude, 36(3), $433-441$.

Costa de Oliveira, L. (2019). Cuidados Paliativos: Por que Precisamos Falar sobre isso? Revista Brasileira de Cancerologia, 65(4), 1-3. https://doi.org/10.32635/2176-9745.rbc.2019v65n4.558

Crenças, A. E., Torno, E. M., Prática, A. S. U. A., Cartagena, E. M., Del, M., Rubio, T., Avila, C. (2017). Representação social dos enfermeiros sobre cuidados paliativos. Revista Cuidarte, 8(1), 1509-1518. http://www.revistacuidarte.org/index.php/cuidarte/article/download/367/759

De Oliveira, M. M., Malta, D. C., Guauche, H., de Moura, L., \& Azevedo \& Silva, G. (2015). Estimativa de pessoas com diagnóstico de câncer no Brasil: Dados da pesquisa nacional de saúde, 2013. Revista Brasileira de Epidemiologia, 18, 146-157. https://doi.org/10.1590/1980-5497201500060013

Ercole, F. F., Melo, L. S. de, \& Alcoforado, C. L. G. C. (2014). Integrative review versus systematic review. Reme: Revista Mineira de Enfermagem, 18(1), 12-14. https://doi.org/10.5935/1415-2762.20140001

Hey, A., Hermann, A. P., Mercês, N. N. A. das, \& Lacerda, M. R. (2017). Participation of Nurses in Palliative Home Care. REME: Revista Mineira de Enfermagem, 21. https://doi.org/10.5935/1415-2762.20170010

Heydari, H., Hojjat-Assari, S., Almasian, M., \& Pirjani, P. (2019). Exploring health care providers' perceptions about home-based palliative care in terminally ill cancer patients. BMC Palliative Care, 18(1), 1-9. https://doi.org/10.1186/s12904-019-0452-3

Instituto Nacional de Câncer José Alencar Gomes da Silva. (2020). ABC do câncer: abordagens básicas para o controle do câncer. https://www.inca.gov.br/sites/ufu.sti.inca.local/files//media/document//livro-abc-6-edicao-2020.pdf

Justino, E. T., Kasper, M., Santos, K. da S., Quaglio, R. de C., \& Fortuna, C. M. (2020). Palliative care in primary health care: Scoping review. Revista LatinoAmericana de Enfermagem, 28, 1-11. https://doi.org/10.1590/1518-8345.3858.3324

Maetens, A., Beernaert, K., De Schreye, R., Faes, K., Annemans, L., Pardon, K., Cohen, J. (2019). Impact of palliative home care support on the quality and costs of care at the end of life: A population-level matched cohort study. BMJ Open, 9(1), 1-9. https://doi.org/10.1136/bmjopen-2018-025180

Malta, D. C., Bernal, R. T. I., Lima, M. G., Araújo, S. S. C. de., Silva, M. M. A. da., Freitas, M. I. de F., \& Barros, M. B. de A. (2017). Doenças crônicas não transmissíveis e a utilização de serviços de saúde: análise da Pesquisa Nacional de Saúde no Brasil. Rev Saude Publica, 51(1), 1-10. https://doi.org/10.1590/S1518-8787.2017051000090

Malta, D. C., Moura, L. de., Prado, R. R. do., Escalante, J. C., Schmidt, M. I., \& Duncan, B. B. (2014). Mortalidade por doenças crônicas não transmissíveis no Brasil e suas regiões, 2000 a 2011. Epidemiologia e Serviços de Saúde, 23(4), 599-608. https://doi.org/10.5123/s1679-49742014000400002

Marcucci, F. C. I., Perilla, A. B., Brun, M. M., \& Cabrera, M. A. S. (2016). Identificação de pacientes com indicação de Cuidados Paliativos na Estratégia Saúde da Família: estudo exploratório. Cadernos Saúde Coletiva, 24(2), 145-152. https://doi.org/10.1590/1414-462x201600020012

Martins, R. S., Correa Júnior, A. J. S., Santana, M. E. de., \& Santos, L. M. S. dos. (2018). Corporeidade de adoecidos oncológicos em cuidados paliativos domiciliares: a vivência de familiares cuidadores. Revista de Pesquisa: Cuidado é Fundamental Online, 10(2), 423. https://doi.org/10.9789/21755361.2018.v10i2.423-431

Matos, M. R., Muniz, R. M., Viegas, A. D. C., Przylynski, D. S., \& Holz, A. W. (2016). Significado da atenção domiciliar e o momento vivido pelo paciente oncológico em cuidados paliativos. Revista Eletrônica de Enfermagem, 18. https://doi.org/10.5216/ree.v18.35061

Oliveira, M. B. P., Souza, N. R. de., Bushatsky, M., Dâmaso, B. F. R., Bezerra, D. M., \& Brito, J. A. de. (2017). Oncological homecare: family and caregiver perception of palliative care. Escola Anna Nery - Revista de Enfermagem, 21(2), 1-6. https://doi.org/10.5935/1414-8145.20170030

Passarelles, D. M. do A., Rios, A. A., \& Santana, R. F. (2019). Diagnósticos de enfermagem em cuidados paliativos oncológicos: revisão integrativa. Enfermería Global, (55), 590-600. http://dx.doi.org/10.6018/eglobal.18.3.345201

Pereira A. S. et al. (2018). Metodologia da pesquisa científica. [e-book]. Santa Maria. Ed. UAB/NTE/UFSM.: https://repositorio.ufsm.br/bitstream/handle/1/15824/Lic_Computacao_Metodologia-Pesquisa-Cientifica.pdf?sequence=1.

Pilatti, P., Lagni, V. B., Picasso, M. C., Puma, K., Mestriner, R. J. de S., Machado, D. de O., \& Zortéa, K. (2017). Cuidados paliativos oncológicos em um serviço público de atenção domiciliar. Revista Brasileira de Medicina de Família e Comunidade, 12(39), 1-10. https://doi.org/10.5712/rbmfc12(39)1339

Sousa, L. M. M. De., Firmino, C. F., Marques-Vieira, C. M. A., Severino, S. S. P., \& Pestana, H. C. F. C. (2018). Revisões da literatura científica: tipos, métodos e aplicações em enfermagem. Revista Portuguesa de Enfermagem de Reabilitação, 1(1), 45-55. https://doi.org/10.33194/rper.2018.v1.n1.07.4391

Sousa, J. M., \& Alves, E. D. (2015). Competências do enfermeiro para o cuidado paliativo na atenção domiciliar. ACTA Paulista de Enfermagem, 28(3), 264269. //doi.org/10.1590/1982-0194201500044

World Health Organization. (2007). Cancer control: knowledge into action. WHO guide for effective programmes. Palliative care. Cancer Control: Knowledge into Action. WHO Guide for Effective Programmes. Palliative Care. https://www.ncbi.nlm.nih.gov/books/NBK195408/ 